\title{
Biotechnology for formation of aromatic properties of national- foodstuffs on the basis of meat raw material under influence of bacterial crops and chromato-mass-spectrometric analysis of the flavoring components
}

\begin{abstract}
Describes the process of the formation of the organoleptic characteristics of the raw sausage "Brunswick", prepared by the original fermented technology of the VM Gorbatov All-Russian Meat Research Institute in the presence of reactive mixtures of starter cultures Lactobacillus plantarum/Staphylococcus carnosus, and Lactobacillus plantarum/Micrococcus varians. Identified changes in the pool of chemicals that make up the flavor and aroma of the national product, associated with the presence of bacterial cultures and added cardamom and black pepper.
\end{abstract}

Keywords: meat raw materials, chromato-mass-spectrometric, lactobacillus plantarum, black pepper, cardamom, isoleucine

\author{
Volume 3 Issue 4 - 2017 \\ Ivankin AN,' Semenova AA, ${ }^{2}$ Nasonova VV, ${ }^{2}$ \\ Kulikovskii AV, ${ }^{2}$ Vostrikova NL, ${ }^{2}$ Rogatin $\mathrm{AI},{ }^{2}$ \\ Baburina $\mathrm{Ml}^{2}$ \\ 'Department of Chemistry, Bauman Moscow State Technical \\ University, Russia \\ ${ }^{2}$ Department of Analytical Research, the VM Gorbatov All- \\ Russian Meat Research Institute, Russia
}

\author{
Correspondence: Andrew N Ivankin, Department of \\ Chemistry, Bauman Moscow State Technical University, I05005, \\ Moscow, 2nd Bauman Str. 5, Moscow, Russia, \\ Email aivankin@inbox.ru
}

Received: May 24, 2017| Published: July 05, 2017

\section{Introduction}

Aroma is the most important organoleptic characteristic of meat products. Aroma is a human response to a complex of volatile substances contained in the air zone around the product when it is consumed. The complexity of studying the fragrance consists in the fact that it is formed by a huge number of substances of different chemical nature. However, these substances are present in the product as a rule in minimal concentrations, but due to the very low threshold of sensitivity of human receptors, they are felt and have a significant effect on the organoleptic evaluation of the product as a whole.

The process of forming the aroma of raw smoked sausages has been studied by domestic and foreign scientists for decades. ${ }^{1-5}$ Interest in this issue is caused by a number of objective reasons. Firstly, smoked sausages are a product of long-term production with high consumer value. Secondly, their manufacture is a combination of the most complicated microbiological and biochemical processes, including enzymatic, proteolytic and oxidative transformations, the final result of which is still difficult to predict even in the conditions of a wellestablished technological process. Thirdly, changing the perception of the safety and quality of sausages smoked under the influence of new scientific knowledge makes us seek ways to modify technological processes and study their impact on the formation of traditional consumer quality products. Currently, over 400 key compounds are established by scientific research conducted abroad, which determine their taste and aroma of traditional fermented sausages produced in different countries. Moreover, such studies were conducted not only in France, Italy, Spain, Belgium, but also in Portugal, Chile, Mongolia and other countries. ${ }^{6-10}$ One of the most popular fermented products in Russia is the smoked sausage "Braunschweig", the key aromatic compounds of which have not yet been studied by modern chromatographic methods. In the last decade, this sausage is made in industrial enterprises using starter cultures containing staphylococci.

Staphylococci, in particular Staphylococcus carnosus, contribute to the formation of a specific flavor of the product by the transformation of amino acids (including branched chain amino acids-leucine, isoleucine and valine) and free fatty acids. Forming of aroma also depends on the type of sausages and the technology of their production. In the case of rapidly ripening sausages, the use of staphylococci promotes the formation of methyl branched aldehydes. In the case of slowly ripening sausages, the use of staphylococci in large quantities promotes the formation of methyl branched acids and sulfites, and at a low level of their introduction diacetyl and ethyl esters are formed. In addition, the formation of aromatic compounds is influenced by the addition of nitrite, nitrate or ascorbate, the parameters of pre-growing cultures and environmental factors. ${ }^{11-15}$ The use of staphylococci, which produce a large number of aromatic compounds, allows improving the organoleptic qualities of sausages and speeding up the process of fermentation. Micrococci as starting microorganisms are recognized as less effective than staphylococci. However, the use of Staphylococci in starter cultures may be accompanied by a risk of developing enterotoxigenic strains of Staphylococcus aureus due to the inability to conduct effective microbiological monitoring in the presence of a technological related microflora. In this connection, taking into account the influence of the technology of making a particular sausage, the research on the comparative evaluation of the aroma of the traditional smoked sausage "Braunschweig" was of scientific and practical interest when replacing the Staphylococcus strain with a micrococcal strain in the starting culture. 
Recently, studies of spatial formation of materials on the principle of 3D printing have been developed. ${ }^{16-33}$ For computer manufacturing in the space of different objects, information on the component composition of the material is needed. Previous studies have made it possible to determine a limited number of constituent substances of different objects. ${ }^{1-15}$ The capabilities of chromatography-mass spectrometry make it possible to establish an almost complete set of constituent chemical components of objects for various purposes. This information can be used for further copying. The aim of the work is a comparative study of the formation of organoleptic characteristics of raw sausage sausages using staphylococci and micrococci in the starting cultures. The objects of the study were samples of smoked sausage of the "Braunschweigskaya" type, manufactured industrially without introduction of the starting culture (control sample "B"), with the addition of a starting culture containing Lactobacillus plantarum + Staphylococcus carnosus (prototype "A"), Culture containing Lactobacillus plantarum + Micrococcus varians (prototype "B").

Control and prototypes were made from minced meat, consisting of $45 \%$ lean beef, $25 \%$ lean pork and $30 \%$ bacon. Nitrite salt was added in an amount of $3 \%$, glucose $0.3 \%$, cognac $0.25 \%$, black pepper $0.1 \%$, cardamom $0.05 \%$, ascorbic acid $0.05 \%$ to ground weight. One strain of Lactobacillus plantarum and strains of Staphylococcus carnosus and Micrococcus varians were used to make sausage prototypes, which were added at the rate of $1.10^{7} \mathrm{U} / \mathrm{g}$ for the introduction of minced meat. Finished minced meat was stuffed into an artificial sausage "fibrouse" with a diameter of $48 \mathrm{~mm}$, forming loaves with a mass of $300 \mathrm{~g}$. All the samples were subjected to a precipitation at a temperature of $+2,+4^{\circ} \mathrm{C}$ for 24 hours. After settling, the loaves were placed in a climatic chamber and kept at a temperature of $+24,+$ $25.5^{\circ} \mathrm{C}$ and a relative humidity of not more than $95 \%$ until the $\mathrm{pH}$ of the product decreases to not more than 5.2. After that, the humidity in the chamber was reduced to values no higher than $92 \%$, and a phased smoking process, lasting 90 minutes, was started. At the same time, the temperature and humidity in the chamber were gradually reduced, bringing them for 8 days to $14^{\circ} \mathrm{C}$ and $74 \%$, respectively. Then the samples of the sausage sausage were dried at a temperature of 12$14^{\circ} \mathrm{C}$ until the final moisture content of the product reached no higher than $32 \%$.

\section{Discussion}

The analysis of the volatile odor constituents was carried out on a gas chromatograph $7890 \mathrm{~A}$ with a mass-selective detector $5975^{\circ} \mathrm{C}$ VLMSD Agilent Technologies (USA). Extracts (1:1) were extracted with $40 \%$ aqueous ethanol and Folce extracts with chloroform-methanol followed by methylation with a solution of acetyl chloride in methanol. ${ }^{11}$ The calculation of the content of the components (represented by the IUPAC name) with a mass content of more than $0.01 \%$ in the flavor/aroma mixture was carried out using the automatic database for searching and identifying the NIST08 MS Library data with a probability of a peak correlation of more than $65 \%$. The composition of the smell of muscat for chemical components that affect the taste and aroma of the Braunschweig sausage can be represented (\%) by chromatographic mass spectrometry: 3-cyclohexene-1-methanol, alpha, alpha-4-trimethyl acetate 1.52; alpha,alpha-4-trimethyl-3-cyclohexene-1-methanol acetate 9.15 ; 5-(2-propenyl)-1,3-benzodioxole 0.53 ; 5-(2-propenyl)1,3-benzodioxole 5.66; 4-(trimethyl)-1H-pyrazole 0.61 ; 2-methyl2,4-dimethoxybutane $\quad 3.70 ; \quad 3,3,6$-trimethyl-1,4-heptadien-6-ol 7.00; methyl-2,4-dimethoxybutane 5.93; 4-[1,3]dioxolan-2-yl3,4-dimethyl-cyclohex-2-enone 0.49 ; dehydroacetic acid 10.32; $\mathrm{N}, \mathrm{N}$-diethyl-p-nitroaniline $\quad 0.15 ; \quad$ d,1-trans-4-methyl-5-methoxy1 -(1-methoxy-1-isopropyl)-cyclohex-3-ene $\quad 0.03 ; \quad 1,5$-hexadiene, 3-chlor-propane $\quad 0.20 ; 1,2,4 \mathrm{a}, 5,6,8 \mathrm{a}$-hexahydro-4,7-dimethyl-1- (1-methylethyl)-naphthalene $1.48 ; \quad$ 1,3-benzodioxole-4-methoxy6-(2-propenyl)-pentamethylbenzoic acid 17.91; 1,2-dimethoxy4-(2-methoxy-1-pro-penyl)benzene 11.78; bicyclo[5.1.0]octane 0,$05 ; \quad 8$-(1-methylethylidene)-naphthalene 0.19 ; hexanoic acid, 4-methylpentyl-este-furan 0.04 , picolinyl $9 \mathrm{c}, 11 \mathrm{t}, 13 \mathrm{t}$-octadecatrienoate 0.07; furane-2-carboxylic acid, 5-[4-(1,1-dimethylethyl) phenoxymethyl]-1,2-benzenediol 0.48 ; furane-2-carboxylic acid, 5-(1,2,4-triazol-1-ylmethyl)-2-aminoresorcinol $0.40 ; 1,2,4 \mathrm{a}, 5,8,8 \mathrm{a}-$ hexahydro-4,7-dimethyl-1-(1-methylethyl)-naphthalene $\quad 1.31$, 1,2,3-trimethoxy-5-(2-propenyl)-benzene 0.11 , hexobarbital 0.35 ; 1,3-dithiacyclohexane 0.03 ; alantolactone 0.14 ; 3-methoxy-Nphenyl-2-propenamide $0.11 ; 1,2,3,4,4 \mathrm{a}, 5,6,8 \mathrm{a}$-octahydro-7-methyl4-methylene-1-(1-methylethyl)-naphthalene $0.28, \quad \mathrm{~N}-(4,6,7,8-$ tetrahydro-3,13-dimethoxy-4-oxoheptaleno(1,2-f)(1,3) benzodioxol6-yl)acetamide $0.75 ;$ 6,6-dimethoxy-octanoic acid, methyl ester 0.22 ; methyl tetradecanoate 4.31, 1-(1-chloro-2,3-dimethylcyclopropyl)3,3-dimethyl-1-butyne $0.04, \mathrm{~N}$-(2-iodo-4-methylphenyl)-2,2,3,3,3pentafluoro-2-[5-(4-chloro-3-nitrophenyl)-propanamide 3.26 1,8-dioxa-cyclohexadecane-2,10-dion 0.12 ; 3-hexadecanone 0.02 ; 4,7,7-trimethyl-5-(tetrahydropyran-2-yloxy)-bicyclo [2.2.1]heptan2-on 0.07 ; epiglobulol $0.06 ; 2$-[(5-chloropentyl)oxy]tetrahydro- $2 \mathrm{H}-$ pyran $0.04 ; 2$-oxo-1-oxaspiro[4,5]decane-4-carbonitrile 0.15 ; p-(2methylallyl)-phenol 0.05 ; linalool 0.30 ; o-methoxybenzonitrile 0.03 ; 1-methyl-3-ethyladamantane 0.04 ; 7-hexadecenoic acid, methyl ester 0.06; hexadecanoic acid, methyl ester 0.65 ; 4-(dimethoxymethyl)cyclohexene $\quad 0.06 ; \quad 4$-methylene-1-(1-methylethyl)-cyclohexene 0.04; 1-dimethylamino-2-hydroxy-cyclobutene-3,4-dione $\quad 0.04$; 5-aminoindazole 0.03 ; N-chloro-succinimide $0.02 ; 8,8$-dimethoxy2,6-dimethyl-2-octanol $\quad 0.08 ; \quad$ 2-ethyltetrahydro-thiophene 0.03 ; 2-[2-[2-(2-methoxyethoxy)ethoxy]ethoxy]ethyl acetate 0.04; phenylacetic acid, 2-adamantyl ester 0.02; 5-butyl-2pyridinecarboxylic acid $0.02 ; 1,2,15,16$-diepoxyhexadecane 0.02 ; 9-octadecenoic acid (Z)-, methyl ester 0.90; octadecanoic acid, methyl ester 0.29; hexadecanamide 0.03; acetic acid, 2,4-diacetoxy3-methyl-1-(trityloxymethyl)pentyl ester 0.05 ; Z-2-octadecen-1-ol 0.06; 9-octadecenal 0.03; 4-oxo-6-(1-piperidyl)-hexanoic acid 0.47; 7-nonenamide 0.03 ; hexadecanoic acid, 2-hydroxy-, methyl ester 0.06; 9-octadecenal 0.76; 2-heptadecanol 0.25; 7,11-hexadecadienal 0.05; tridecyl-oxirane 0.03 ; 18-pentatriacontanone $0.12 ; 2$, 13-octadecadien1-ol 0.03; octadecanal 0.51; 1-methyl-cyclopentanol 0.14.

The chemical composition of the components of the smell of the second spice - black pepper, which is used in the recipe of the sausage Braunschweigskaya can be written down (\%):9-octadecene 0.01 ; 3-undecen-1-yne 0.01; (+)-4-carene 2.64; 1,2,6,6-tetramethyl1,3-cyclohexadiene $\quad 0.01 ; \quad 1$-methyl-4-(1-methylethylidene)cyclohexene 0.02 ; alpha-phellandrene 0.05 ; 1S-alpha-pinene 0.03 ; 1 -methyl-4-(1-methylethyl)-benzene 0.68 ; limonene 0.27 ; tetrahydro2,2-dimethyl-5-(1-methyl-1-propenyl)-furan $\quad 0.01 ; \quad$ 4-ethenyl1,5,5-trimethyl-cyclopentene 0.01 ; 3-carene 1.47; alpha-fenchylmethylether $0.29 ; \quad 1$-methyl-4-(1-methylethylidene)-cyclohexene $1.72 ; \quad 2$-methylisoborneol $\quad 0.06 ; \quad 3,7,7$-trimethyl-bicyclo[4.1.0] hept-2-ene 0.65 ; 2,7-dimethyl-1,3,7-octatriene 0.24 ; 1-methyl-4-(1methylethyl)-3-cyclohexen-1-ol $0.03 ; 2,6$-dimethyl-2,4,6-octatriene 0.09 ; camphene 0.03 ; ocimene 0.55 ; N-methyl-N-hexadecylethylamine $1.23 ; \quad 1,1$-dimethyl-2-(3-methyl-1,3-butadienyl)cyclopropane 0.03 ; biperiden 0.02 ; N-methyl-1H-purin-6-amine 0.19 ; 3-methoxy-3-methyl-1-pentene $\quad 0.08 ; \quad$ 2-(6-heptynyl)-1,3dioxolane 0.05 ; alpha,alpha-4-trimethyl-3-cyclohexene-1-methanol acetate $1.01 ; 1$-methyl-4-(1-methylethylidene)-cyclohexanol 0.84 ; 2-methylene-5-(1-methylethyl)-cyclohexanone $\quad 0.18 ; \quad 3,5$-bis1-dimethylethyl)-4-hydroxy-2,4-cyclohexadien-1-one 0.04 ; octahydro-spiro[1,3-dioxolane-2,1'(4'H)-naphthalen]-4'-one 0.02 ; caryophyllene $0.06 ; 2$-undecanone $0.01 ; 7$-amino-3-ethyl-4(3H)- 

components

quinazolinone $\quad 0.07 ; \quad 4$-aminoresorcinol $\quad 0.07 ; \quad 2,3$-dimethyl-3buten-2-ol $\quad 0.05 ; \quad 1$-methyl-4-(1-methylethyl)-1,3-cyclohexadiene 0.57 ; 7-methoxy-3,7-dimethyl-octanal $1.16 ;$ d,1-trans-4-methyl5-methoxy-1-(1-methoxy-1-isopropyl) cyclohex-3-ene 0.68 ; 1-chloro-3-iodo-cycloheptane 0.28; tricyclo[7.2.0.0(3,8)] undec-4ene 3.34 ; 4-dimethylaminopyridin-2-amine $0.42 ; 1,2,3,4,4 \mathrm{a}, 5,6,8 \mathrm{a}-$ octahydro-7-methyl-4-methylene-1-(1-methylethyl)-naphthalene $2.10 ; \quad 1 \mathrm{~S}, 2 \mathrm{~S}, 5 \mathrm{R}-1,4,4$-trimethyl-tricyclo[6.3.1.0(2,5)] dodec-8(9)ene $0.05 ; 1,2,4,8$-tetramethylbicyclo[6.3.0]undeca-2,4-diene 0.36 ; 6-ethenyl-6-methyl-1-(1-methylethyl)-3-(1-methylethylidene)cyclohexene $\quad 0.18 ; \quad 1,2,3,6,7,7 \mathrm{a}$-hexa-hydro-2,2,4,7a-tetramethyl1,3a-ethano-3aH-indene 1.39; 1,2,3,5,6,8a-hexahydro-4,7dimethyl-1-(1-methylethyl)-naphthalene $\quad 0.14 ; \quad 1$-ethyl-1-phenylhydrazine $\quad 0.65 ; \quad 1,2,4 \mathrm{a}, 5,8,8 \mathrm{a}-\mathrm{h}$ exahydro-4,7-di-methyl-1-(1methylethyl)-naphthalene $2.11 ; 3,5$-dimethyl-benzenemethanol 0.10 ; 1,2,3,5,6,8a-hexa-hydro-4,7-dimethyl-1-(1-methylethyl)-naphthalene 0.85 ; copaene 0.08 ; alpha-calacorene $0.10 ; 2,6,10$-trimethyl-3-oxo12-(tetrahydropyran-2-yloxy)-dodeca-6,10-dien $\quad 0.12$; decahydro4a-methyl-1-methylene-7-(1-methylethenyl)-naphthalene 0.33 ; cholestan-22(26)-isoepoxy-3-beta-ol $1.60 ; \quad 6,6$-di-methyl-10methylene-1-oxa-spiro[4.5]decane 0.56 ; 4-Aminoresorcinol $9.20 ; \quad 2,4,6$-pyrimidinetriamine $\quad 5.90 ; \quad 1,2,4 \mathrm{a}, 5,8,8 \mathrm{a}$-hexahydro4,7-dimethyl-1-(1-methylethyl)-naphthalene 2.61; 6-ethyl-2phenyl-indolizine $2.82 ; \quad \mathrm{N}$-(4-methoxyphenyl)-2,2-dimethylpropanamide $\quad 0.83 ; \quad 2,3,4,7,8,8 \mathrm{a}$-hexahydro-3,6,8,8-tetramethyl$1 \mathrm{H}-3 \mathrm{a}, 7$-methanoazulene 0.97 ; guaia-3,9-diene $0.50 ; 1,2,3,4,4 \mathrm{a}, 7-$ hexahydro-1,6-dimethyl-4-(1-methylethyl)-naphthalene $\quad 0.50$; 1,5,5-trimethyl-6-methylene-cyclo-hexene $\quad 0.15$; himachala2,4-diene $\quad 0.38 ; \quad 3,7,7$-trimethyl-bicyclo[4.1.0]hept-3-ene 1.03; 1-(1-chloro-2,3-dimethylcyclopropyl)-3,3-dimethyl-1-butyne 3.06; 4-(1,2-dimethyl-cyclopent-2-enyl)-butan-2-one 0.37 ; decahydro1,1,7-trimethyl-4-methylene-1H-cycloprop[e]azulene 0.21 ; 1,1a,5,6,7,8-hexahydro-4a,8,8-trimethyl-cyclopropa[d]naphthalen2(4aH)-one $\quad 0.16 ; 1$-ethyl-cyclohexanol $0.38 ; 11$-methylene-2,4- dimethyl-3-azatricyclo[5.3.1.0(4,9)]undec-2-ene 0.48 ; decahydro1,1,7-trimethyl-4-methylene-1H-cycloprop[e]azulene $\quad 1.05$; 3,3-dimethyl-2-(3-methyl-1-butenyl)-cyclohexanemethanol 0.20 ; 1S,2S,5R-1,4,4-trimethyltricyclo[6.3.1.0(2,5)] dodec-8(9)-ene 0.36; 7-hexadecenoic acid, methyl ester 0.15; hexadecanoic acid, methyl ester 1.15 ; 2,3-epoxy-geranyl acetate 0.01 ; 5-methylnonane 0.15 ; undec-2-enyl ester valeric acid 0.04; 6,6-dimethoxyoctanoic acid, methyl ester 0.04 ; 2-[[(5-alpha,17-beta)-androstan17-yl]oxy]tetrahydro-2H-pyran $0.07 ; \quad 1,13$-tetradecadiene 0.03 ; 9-octadecenoic acid (Z)-, methyl ester 2.35; octadecanoic acid, methyl ester 0.47 ; octadecanedioic acid 0.04 ; palmitic acid vinyl ester $0.42 ; 7,11$-hexadecadienal $0.38 ; 2$-dodecylcyclobutanone 5.65 ; 9-Octadecenamide, (Z)- 0.05; 5-amino-1H-tetrazole-1-ethanol 0.07; 9-octadecenal, (Z)- 9.14; 3-methoxymethoxy-2,3-dimethylundec-1ene 4.45 ; methyl-10-trans,12-cis-octadecadienoate 0.16 ; decanoic acid, cyclohexyl ester 3.68; 2-nonyl-cyclopropaneundecanal 2.26; decyl sulfide 0.45 ; 1-hexadecyne $4.79 ; 2$-methyl-hexadecanal 1.60; 4-methyl-heptadecane 0.24 .

One would expect that the substances of these spices will constitute the main bouquet of the taste of sausages. However, as can be seen from Table 1, the initial stuffing and cooking from it by accelerated fermentation of the sausage with introduced crops have an original composition of the basic substances that determine exactly the aroma of the sausage "Braunschweig". From the list of substances presented in Table 1 it can be seen that some substances or their derivatives characteristic for the organoleptic components of muscat and black pepper used in the recipe of the Braunschweigskaya sausages are present in the final product, however, the pool of substances that is ultimately formed under the action of starter cultures and technological operations eventually turns out to be practically Unique. It is known that many organic substances have a characteristic flavor, for example, indolizin-cucumber, 2-nonenal-watermelon, tetradecanoic acidbalsam, incense, 1-octen-3-ol-mushrooms, n-decane and guaiacoltobacco, indole-fruits Pendadecanal - burnt wood, etc. ${ }^{12}$

Table I Composition of flavoring components in fat-free samples, $\mu \mathrm{g} / \mathrm{kg}$

\begin{abstract}
IA
Cyclopentyl ester 2.82; I0-pentadecen-5-yn-I-ol 0.85; 6-tetra-O-methyl-octanoic acid; ethyl ester 0.89; trenbolone 0.93; 2-ethylacridine 2.3I; eicosane 2.I4; pyridine 2.26; I0-methylnonadecane 4.0I; 5,6-dimethyl-phenanthridinium 2.22; I-methyl-4-(I-methylethyl)-I,3-cyclohexadiene 0.94; I-undecene I.7I; I-phenyl-4-(2-cyano2-phenylethenyl)benzene 0.54 ; heneicosane 3.62; 3,5-bis(I,I-dimethylethyl) 0.93 ; tetratriacontane 2.47; 2-hexen-I-ol 0.3I; 7-methoxy-3,7-dimethyl-octanal 0.56; triacontane 3.37; (3s)-pentanol I.02; I-pentadecene 0.33; I-dodecene 0.67; 4-methyl-2-hexanone 0.34; tetratriacontane 2.6I docosane I.I3; ethyl ester decanoic acid 3.86; n-nonadecanol-I 7.53; 3-methyl-tridecane 0.94; 5-ethyl-2-methyl-octane 7.|3; octadecane 2.I6; 2-naphthyl-p-tolyl sulfone 0.40; N-(2'-acetyl-4',5'dimethoxyphenyl)-4-methoxy-benzamide 0.21 ; trans-2,3-methylenedioxy-b-methyl-b-nitrostyrene 0.29 ; (3,4-dimethoxy-benzyl)-(4-morpholin-4-yl-phenyl)-amine 0.46; N-(2-chloroethoxycarbonyl)-l-methionine, propyl ester 0.45; nexahydro-2H-pyrido(I,2-a)pyrazin-3(4H)-one 0.69; d-gluco-d-gulo-heptose, diethyl mercaptal 0.27; I-(2-hydroxy-5-methylphenyl)-I-octanone (E)-oxime 0.I4; 2,3-dihydro-2,8-dimethyl-benz[b]-I,4-oxazepine-4(5H)-thione 0.55; 5-(4-ethoxyphenyl)-3-(4-pyrrolI-ylphenyl)-[I,2,4] oxadiazole 0.4I; 2-methylaminomethyl-I,3-dioxolane 0.36; I-acetyl-4-[I-piperidyl]-2-butynone 0.35; ethanethioic acid 0.2 I; S-[8-(diethylphosphono) octyl] ester $0.1 \mathrm{I}$; paroxetine $0.4 \mathrm{I} ; 2,4,6$-trichloro-5-nitro-pyridine-3-carboxamide 0.15 ; I,2,3,6-tetrahydro-I-methyl-4-[4-chlorophenyl]-pyridine 0.08; 8,8'-dimethyl2,2'-binaphthalene-I,I',4,4'-tetrone 0.47; 2-penta-2,4-dienyl-cyclohexanecarboxylic acid, methyl ester 0.33; I-(4-pyridinyl)-ethanone, oxime 0.26; N,N-dimethylphosphoramidocyanidic acid, methyl ester 0.47; 2,5-diphenyl-oxazole 0.56; 2-amino-I-piperidin-I-yl-propan-I-one 0.26; N-benzyl-N-ethyl-p-isopropylbenzamide 0.72; 6-chloro-I-nitro-naphthalene 0.45 ; 8-(3-ethoxypropylamino)-I,3-dimethyl-3,9-dihydro-purine-2,6-dione 0.29; 2,2,5-trimethyl-6-trimethylsilylmethylenecyclohex4-ene-I,3-dione $\quad 0.25 ; \quad 6,7$-dimethoxy-3-[2-(2-methoxyphenyl)-2-oxoethyl]-I(3H)-isobenzofuranone $\quad 0.18 ; \quad 2-[(\mathrm{N}, \mathrm{N}-$ dimethylamino)methyl]-3,5-dimethyl-phenol 0.26; 3-(3,6-dimethylocta-2,7-dienyl)-IH-indole 0.12; 2-acetyl-3-methoxy-benzeneacetic acid 0.7I; chlorthiophos 0.3I; 3-methylbenzothiophene 0.26; 3-amino7-nitro-I,2,4-benzotriazine-I-oxide 0.10 ; N-(cyclohexylcarbonyl)-sarcosine, nonyl ester 0.27; I-(3-methoxy-phenyl)-5-(5-methyl-furan-2-ylmethylene)-2-thioxodihyd $\quad 0.37$; 6 -nitro-2-p-tolyl-2H-indazole $0.24 ; \quad 2$-fluoro-acetamide 0.28 ; (3,5-dimethyl-piperidin-I-yl)-(2-iodo-phenyl)-methanone 0.27 ; $5 \mathrm{H}$-thiazolo[2,3-a] pyridine-8-carboxamide 0.15 ; 3 -(I-adamanthyl)-7-methyl 0.26 ; 2,5-dimethylbenzoxazole 0.25 ; 2-chloro-4-quinolinecarboxylic acid 0.28 ; $\mathrm{NI}$-phenethyl-2-(I,3benzodioxol-5-ylmethylidene)hydrazine-I-carbothioamide 0.62 ; 4-butyloxy-2-hydroxybenzamidine 0.57 ; 4,6-bis(diethylamino)-I,3,5-triazine-2-carbonylhydrazide 0.33; 2-ethylacridine 0.24 ; N-methyl-I,3-propanediamine 0.18 ; octahydro-2-(4-methoxyphenyl)-2H-I,3-benzoxazine 0.16 ; 4-[5-(diethylaminosulfonyl)-2-(2-furyl)I-benzimidazolyl]butyric acid 0.06; 6,7-dimethoxy-3-[2-(2-methoxyphenyl)-2-oxoethyl]-I(3H)-isobenzofuranone 0.17; 2-(5-bromopentyl)-2-(phenylmethyl)-I,3dithiane 0.0I; 9-[3-(dimethylamino)propyl]-9-Borabicyclo[3.3.I]nonane 0.38; 2,6-pyridine diamidoxime 0.46; 5-(4-methyl-I,3-dioxolan-2-yl)-I,3-benzodioxole 0.27; 2-(acetoxymethyl)-3-methoxycarbonyl)biphenylene 0.24; 2-(acetoxymethyl)-3-(methoxycarbonyl)biphenylene 0.42; 2-(methylamino)-ethanol 0.16; 2-amino-acetamide 0.23; 4-bromo-3-chloroaniline 0.25; I-[2-[4-(I-methylethyl)phenyl]-4-nitro-I,3-dioxan-5-yl]-methanol 0.3I; 3-ethoxy-4-methoxyphenylacetonitrile 0.03; acetamide 0.16 ; chlorthiophos 0.15 ; 9, I0-(I,2-benzeno)anthracene, 2,3-dimethyl-9, I0-dihydro-IH-indole-2,3-dione 0.25 ; ethanone 0.22 ; 4H-I-benzopyran-4-one 0.23 ; 4-acetyl5-(2-fluorophenyl)-I,5-dihydro-3-hydroxy-I-methyl-2H-pyrrol-2-one 0.5I; 2-propoxy-ethanamine 0.19; 3-phenyl-2H-chromene 0.20; tocainide 0.I5; 3-[(4-nitrophenyl) methyl]-I,2,3-benzotriazin-4(3H)-one 0.20; acridine-9-carbaldehyde 0.42; IH,4H-benz[d]-I,3-thiazin-2-one 0.23; (9-oxo-9,10-dihydroacridin-4-yl)acetic acid 0.10; 2-(methylamino)-ethanol 0.15 ; 6-methyl-2-phenyl-IH-indole $0.8 \mathrm{I}$; I-anthracenyl-2-pyridyl ketone 0.38 ; 4-phenyl-3,4-dihydroisoquinoline 0.27 ; trans-4'-(methylthio) chalcone 0.2I; N-isopropyl-3-phenylpropanamide 0.13; 4-methyl-2--(4-isopropylbenzylidenamino)-phenol 0.II; 2-ethyl-acridone 0.09; I,3,4,5,6-pentamethyl-2(IH)pyridinone 0.12; 2,3-dibromo-2,3-dimethylbutane 0.35; 2-chloro-4-trifluoromethyl-benzamide 0.04; N-(aminocarbonyl)-2-ethyl-2-butenamide 0.27; N,N,N'-triethyl-I,4benzenediamine 0.27; 4-bromo-2-chloro-benzenamine 0.27; 9-amino-I,8-dimethyl-3,6-diazahomoadamantane 0.I I; methyl 3-(I-pyrrolo)thiophene-2-carboxylate 0.20; 2,5-dimethoxycinnamic acid $0.1 \mathrm{I} ; \mathrm{I}, 2,5 \mathrm{a}, 6,7,8$-hexahydro-6,6-dimethyl-cyclopenta[c]pentalen-3(3aH)-one 0.I2; 2-(3-methylphenyl)-I-phenyl-I-propene 0.I5.
\end{abstract}

Citation: Ivankin AN, Semenova AA, Nasonova VV, et al. Biotechnology for formation of aromatic properties of national- foodstuffs on the basis of meat raw material under influence of bacterial crops and chromato-mass-spectrometric analysis of the flavoring components. J Appl Biotechnol Bioeng. 20I7;3(4):366-372. DOI: I0.15406/jabb.2017.03.00072 
Table Continued. IB

4-ethylbenzamide 4.36; alpha-[2-(dimethylamino)propyl]-alpha-phenyl-benzeneacetonitrile I.37; 4-ethylbenzoic acid, 2-methylbutyl ester 4.20; methoxy-phenyl-oxime 3.3I; 4-morpholin-4-yl-benzaldehyde 2.4I; 2-amino-4-methyl-I-pentanol 0.4I; dimethylaminomethyl-isopropyl-sulfide 3.26; ornithine I6.33; 3-methyl-phenol 6.69; I,2-dimethyl-piperidine 2.04; I,4-octadiene 3.I6; 8-azaguanine 0.97; 2,5-dimethoxy-benzaldehyde 2.30; N-methyl-p-acetophenetidide I.64; dimethyl-phosphoramidous difluoride 4.93; 2-methyl-bicyclo[2.2.2] octan-I-ol I.00; 3-amino-2-cyclohexenone 3.02; 2-nonadecanone 0.90; hexadecanoic acid, methyl ester 2.I6; hexadecanoic acid, ethyl ester 5.85; 2-heptadecanone 1.20; methyl-16-methyl-heptadecanoate II.25; E-I,9-hexadecadiene I.88; 9-octadecenamide 3.86; hex-4-ynoic acid, methyl ester 0.52; N,N-di-2-propynyl-2-propyn-I-amine 0.2I; 3-hydroxy-5-phenyl-I,3-dihydro-benzo[e][1,4]diazepin-2-one 0.38; 3,3,3-trifluoropropanoate 0.45; N-(2'-acetyl-4',5'dimethoxyphenyl)-4-methoxy-benzamide $0.26 ; \mathrm{N}$-(4-chlorophenyl)-carbamic acid, 4-nitrophenyl ester 0.17 ; 2,4-hexadienedioic acid 0.16; ethyl tribromopyruvate $0.15 ;$ I,5-dibromoadamantane-2,6-dione bis(ethylene ketal) 0.23; 4,4a,5,6,7,8-hexahydro-4,4a-dimethyl-6-(I-methylethenyl)-2(3H)-naphthalenone 0.I7; I,I,3,3-tetraallyl-I,3disilacyclobutane 0.48 ; 4-(dimethylamino)-ethoxy-3-buten-2-one 0.48; (2-pent-2-enylcyclopentylideneaminooxy)acetic acid, methyl ester 0.26; 2-amino-5-methyl-[I,2,4] triazolo[I,5-a]pyrimidin-7-ol $\quad 0.14 ; 2$-methoxy-I-(phenylmethoxy)-4-(I-propenyl)-benzene 0.21 ; 2-methyl-furan 0.17; 3-nonynoic acid 0.22; 3,5-cyclohexadieneI,2-dione 0.19; 2-phenoxyphenylacetonitrile 0.22; 4-carboxy-N,N,N-trimethyl-benzenaminium 0.I2; I, I,3,3,5,5,7,7,9,9-decamethyl-carbonic acid 0.I8; 5-(2-nitro-Ipropenyl)-I,3-benzodioxole 0.17; o-anisaldehyde 0.11 ; nonadecylamine 0.26; 2,4-dioxo-3-pyrrolidinecarboxylic acid, methyl ester 0.40; 2,4-di-tert-butylthiophenol 0.38; ethylphenylmalonic acid diethyl ester 0.23; 9-hydroxy-2-methyl-8-nitro-pyrido[I,2-a]pyrimidin-4(5H)-one 0.I5; I,8-diamino-3,6-dioxaoctane 0.08; 2-acetylamino3-(4-ethoxy-phenyl)-acrylic acid 0.30; (methylenedi-4,I-phenylene)bis-carbamic acid, diethyl ester 0.07; fumaric acid, 3-pentyl undecyl ester 0.08; 5-acetamido-4,7dioxo-4,7-dihydro enzofurazan 0.08; 3-hydroxy-5,7-dimethoxy-2-(4-methoxyphenyl)-4H-I-benzopyran-4-one 0.20; N-(2-ethylphenyl)-carbonic acid, monoamide, propyl ester 0.22; 4-bromo-2-chloro-benzenamine 0.18; 2-furanmethanol 0.06; I,I-cyclopropanedicarboxamide 0.24; t-butyl cyclopentaneperoxycarboxylate 0.08; 6-amino-4-hydrazino-IH-pyrazolo[3,4-d]pyrimidine 0.09; 2-chloro-4-trifluoromethyl-benzamide 0.14; N,N'-diethyl-urea 0.19; 2-hexyne 0.27; bifenox 0.09; 4-Bromo-3chloroaniline 0.05 ; 2-isopropylbenzaldehyde 0.23 ; acryl glycine 0.1 I; 2-[4-(I-oxo-3-phenyl-2-propenyl)phenyloxy]-acetamide 0.27; I-methyl-2-propenyl-3-ol benzene 0.14 ; 2-methyl-I-nitro-9, I0-anthracenedione 0.04; 4-(dichloromethyl)-5-chloro-2H)-furanone 0.1 I ; ethyl tribromopyruvate 0.16 ; 2-ethylacridine 0.26 ; nonadecylamine 0.16; $\mathrm{N}$-(diphenylethenylidene)-methanamine 0.14; p-(9-acridinylamino)phenyl-acetic acid 0.19; 4,6-bis(I,I-dimethylethyl)-3-nitro-3,5-cyclohexadiene-I,2-dione 0.35; I-arabinose, di(thiononyl-) acetal 0.19;3,4-diethoxy-beta-methyl-beta-nitrostyrene 0.12;2-nitro-4-(trifluoromethyl)phenol 0.19; trans-3-ethoxy-b-methyl-b-nitrostyrene 0.15 ; tert-butyl 2-aminophenylcarbamate 0.16 ; 3-nitrophthalhydrazide 0.25 ; I-octadecanamine 0.17 ; tetrahydro-6a-methylimidazo[4,5-d]imidazole-2,5(IH,3H)dione 0.08; 9-(4-butyl-benzyl)-acridine 0.17; trans-2,3-methylenedioxy-b-methyl-b-nitrostyrene 0.20; 2-(benzthiazol-2-yl)-6-methoxybenzofuran 0.09; 2-mercaptobenzothiazole-6-carboxylic acid 0.1 I ; bifemelane 0.10;2-acetylamino-3-(4-ethoxy-phenyl)-acrylic acid 0.06;2-methylene-butanediamide 0.15; hexamethylphosphoramide 0.06; 4-fluoro-3-nitrobenzotrifluoride 0.08; (ethenylthio)-benzene 0.13; N-(6-acetylaminobenzothiazol-2-yl)-2-(adamantan-I-yl)-acetamide 0.20; I-benzo[I,3]dioxol-5ylmethyl-6,7-dimethoxy-2-nitroso-I,2,3,4-tetrahydroisoquinolin-4-ol 0.03; sucrose 0.22 ; $\mathrm{N}$-(2-ethylphenyl)-carbonic acid, monoamide 0.07 ; I-heptadecanamine 0.08 ; phenylimine 0.25;4-(I-methylethyl)-benzaldehyde 0.05;3-nitrophthalhydrazide 0. I 3;2-tert-butylaminomethylene-5,5-dimethyl-I,3-cyclohexanedione 0.3 I; 3,3-diphenylacrylophenone 0.24; 2-propyl-2-heptenal 0.48; 3-phenyl-2-propenoic acid 0.18; 4-acetyl-benzoic acid 0.36; styracitol 0.22.

IC

4-ethylbenzamide 3.89; methoxy-phenyl-oxime I.79; D,L-valine 0.59; 4-(I,I-dimethylethyl)-benzenemethanol I.50; 4-ethylbenzoic acid, undecyl ester 4.I0; 2-methyl2-propenoic acid 0.82; 2-ethylacridine 2.46; 2-methyl-2-pentenal 2.3I; ornithine 5.37; bicyclo[4.I.0]heptan-3-ol 2.92; 2-methyl-cyclopentanone I.52; 3-methyl-phenol 3.95; 2,3,5-trimethyl-I,4-benzenediol 0.89; II,I I-dimethyl-bicyclo[8.2.0]dodecane 1.37; 4-(2-aminophenylthio)-5-methyltetrahydrofuran-2-one 4.I5; 3-methoxy-2,4,6trimethyl-benzenamine 0.42; 8-azahypoxanthine I.94; 6-methyl-2-propyl-4(IH)-pyrimidinone 0.58; triacontane I.58; II-(I-ethylpropyl)-heneicosane 4.0 I; tetradecanoic acid, ethyl ester 2.92; azacyclotridecan-2-one 0.54; 2-nonadecanone I.36; I4-methyl-pentadecanoic acid, methyl ester 2.7I; hexadecanoic acid, ethyl ester 7.08 I-acetoxynonadecane 2.58; 2-nonadecanone I.44; octadecanoic acid, methyl ester I.07; trans-I3-octadecenoic acid 5.88; octanamide 3.98; 2,3-2H-4-methyl-imidazole2-one 0.92; 9-octadecenamide I.50;4-[p-chlorobenzyl]-I -methylpiperidine 0.67;2-[4-(2-hydroxyethylamino)-2-quinazolinyl]-phenol 0.33; 4-iodo-N-(4-pyridinylmethyl)IH-pyrazole-I-acetamide 0.39; 3-[I-[4-[I-(2-cyanoethoxy)cyclohexyl]buta-I,3-diynyl]cyclohexyloxy]-propionitrile 0.17; 3-(4-methylphenyl)-I-phenyl-2-propen-I-one 0.36; 2-nitrobenzyl bromide 0.13 ; 3-(2-methoxy-5-methylphenyl)propionic acid 0.12; I-(3,4-methylenedioxyphenyl)-2-propanone-2-oxime 0.22; acridine-9-carbaldehyde 0.22; propanamide 0.13 ; 3-heptyn-I-ol $0.1 \mathrm{I}$; 2-amino-4-hydroxy-6,7-dibenzylpteridine 0.55 ; I,2-bis(I-methyl-3,6-diazahomoadamantantylidene-9)hydrazine 0.24 ; 6,8,9-trichloro-I,4-dioxa-spiro[4.4]non-8-en-7-ol 0.I5; 3,5-dibromo-4-pyridinol 0.10; 4-(4-methyl-I-piperidyl)-5-spiro-cyclohexane-furan-2(5H)-one 0.25; 6-methoxy$\mathrm{N}$-(4-methylbenzylideno)-benzothiazol-2-amine 0.40; N-(3-chlorophenyl)maleimide 0.27; 2-(adamantan-I-yl)-N-adamantan-I-ylethyl)-acetamide 0.23; alpha-methylenebenzeneacetic acid 0.26; I-methylcholanthrene 0.I5; (Z)-2-methoxymethoxy-4'-methyl-stilbene 0.33; paroxetine 0.09; diethyl[I-ethyl-2-(trimethylstannyl)-I-propenyl]borane 0.23 ; 4-(2-hydroxyethylamino)-I-oxo-2-phenyl-I,2-dihydro-phthalazine 0.21 ; 3,6-dimethoxy-9-(2-phenylethynyl)-fluoren-9-ol 0.27; N-[2-(acetyloxy)-2-[4(acetyloxy)-3-methoxyphenyl] ethyl]-acetamide 0.I5; N-(aminocarbonyl)-2-ethyl-2-butenamide 0.25; 4-iodo- $\mathrm{N}$-(phenylmethyl)- I H-pyrazole-I-acetamide 0.33; 2-methyl6-(5-methyl-2-thiazolin-2-ylamino)pyridine; 2-chloro-4-trifluoromethyl-benzamide 0.58 ; n-hexadecylsuccinic anhydride 0.07 ; 2,3-dihydroxy-6-nitroquinoxaline 0.20 ; 4-dehydroxy-N-(4,5-methylenedioxy-2-nitrobenzylidene)tyramine 0.06; carbamic acid, methyl ester 0.39; 4-butyloxy-2-hydroxybenzamidine 0.I 0; I,2-bis(trimethylsilyl) benzene 0.20; I-octadecanamine 0.50; 6,7-dimethoxy-3-[2-(2-methoxyphenyl)-2-oxoethyl]-I (3H)-isobenzofuranone 0.I I; 3-amino-2-phenazinol 0.04; 5-[(acetyloxy) methyl]-3a,4,6a,7,9, I0, I 0a, I0b-octahydro-3a, I0a-dihydroxy-2, I0-dimethyl-benz[e]azulene-3,8-dione 0.09; 3-alpha, I2-beta-dihydroxy-bisnor-5,7-cholenic acid 0.06; bromo-benzene 0.09; $\mathrm{N}$-methyl-anthranilic acid, butyl ester 0.10; dodecahydropyrido[I,2-b]isoquinolin-6-one 0.26; chlorthiophos 0.13; 2-(3,3-dimethyl-but-I-ynyl)I,I-dimethyl-3-methylene-cyclopropane 0.16 ; 9-[3-(dimethylamino)propyl]-9-borabicyclo[3.3.I]nonane 0.28 ; 4-phenyl-pyrido[2,3-d]pyrimidine 0.3 I; 2-amino-Ipiperidin-I-yl-propan-I-one 0.07; 3-(dimethylamino)-I-(2-furyl)prop-2-en-I-one 0.30; I-ethynylcyclopentanol 0.13; 5-(IH-benzoimidazol-2-ylmethylene)-I,3-diethyl2-thioxo-dihydropyrimidine-4,6-dione $0.1 \mathrm{I} ;$ 4-(3-hydroxy-2,6,6-trimethylcyclohex-I-enyl)pent-3-en-2-one 0.13; 4-acetyl-I-(2-furfuryl)-3-hydroxy-5-(4-nitrophenyl)pyrrol-2(5H)-one 0.II; guaifenesin di-tms derivative 0.20; I-[I-(2-fluorenyl)ethylidene]semicarbazide $0.1 \mathrm{I}$; (I-ethoxyethenyl)-benzene 0.12 ; 4-phenyl-pyrido[2,3-d] pyrimidine 0.26 ; 2,2'-bipyridine 0.03 ; methylene-propanedinitrile 0.15 ; nonadecylamine 0.31 ; 2-ethyl-4,5-diphenyl-oxazole 0.24 ; cis-2,3,4-trimethoxy-beta-methyl-betanitrostyrene 0.14 ; 4-allyl-3-(3-furyl)- $\mathrm{IH}$-I,2,4-triazole-5(4H)-thione 0.24 ; 3,5-dibromo-4-pyridinol 0.40; $\mathrm{N}$-methyl-I-adamantaneacetamide 0.17; 2-amino-4-hydroxy6,7-dibenzylpteridine 0.05 ; 6,7,8,9-tetrahydro-3-(2-pyridyl)-pirimido[4,5-b]benzothiophen-I(2H)-one 0.05 ; 4-phenyl-3,4-dihydro-soquinoline $0.1 \mathrm{I}$; 2-methyl-5-(4morpholinyl)-cyclohexa-2,5-diene-I,4-dione 0.15 ; 2-tert-butylamino-6-chloro-4-cyclohexylamino-1,3,5-triazine 0.05; 5-acetamido-4,7-dioxo-4,7-dihydrobenzofurazan 0.1 I; 2,4,6-trifluoronitrobenzene 0.1 3; I-piperonyl-3-sulfamoyl-I,2,4-triazole 0.22; 2-amino-3-cyano-5-aldoximinopyrazine-I-oxide 0.08; 3-chloro-2,3-dihydroximinopropanenitrile $0.11 ; 2,4^{\prime}$-bipyridine 0.15 ;atomoxetine $0.10 ; 1,1,2,2,3,3$-hexamethyltrigermane $0.04 ; 4$-methoxymethylbenzamide $0.04 ; 2$-allylaminomethylene-5,5-dimethylcyclohexane-I,3-dione 0.01 ; I,5-dibromo-hexane 0.02 ; 5-acetylsalicylamide 0.08 ; 4-bromo-2,3,5,6-tetrafluorobenzonitrile 0.43 ; 2-(4-chloro-phenyl)-8,8-dimethyl-9dihydro-7H-pyrazolo[I,5-a]quinazolin-6-one 0.26; 3-phenyl-2-propenoic acid 0.I3; 2-(3,4-dimethoxyphenyl)-3,7-dimethoxy-4H-I-benzopyran-4-one 0.19; $\mathrm{N}$-octyl- $\mathrm{N}$ sec-butyl-3-(2-hydroxy-3,4-dimethyl-phenyl)-3-phenyl-propionamide 0.04; nonylamine 0.08; propanedinitrile 0. 10; 6-bromo-4-phenyl-quinazoline-2-carbaldehyde oxime $0.1 ; \mathrm{N}$-(2-phenylbenzimidazol-6-yl)-acetamide 0.16;2-butyl-4,5,6,7-tetrahydro-IH-isoindole-I,3(2H)-dione 0.1 6; 2,3-dihydro-7-hydroxy-2,2-dimethyl-4H-I-benzopyran4-one 0.02; 3-phenyl-I,2,4-thiadiazol-5-amine 0.08; 4-[(2-hydroxy-5-nitrophenyl)methylenamino]-benzophenone 0.19; (2-bromo-3-methylphenyl)diphenylphosphine 0.01; benzo[I,2,5]oxadiazol-5-ylamine 0.13; 4-amino-2-nitro-phenol 0.13; paroxetine 0.14; NI-[4-(trifluoromethoxy)phenyl]-2-chloroacetamide 0.27; 2-bromo-Nmethyl-2-propen-I-amine 0.I0; 4-acetamido-N,N-dimethyl-3-nitrobenzamide 0.32; N-methyl-I-adamantaneacetamide 0.12 ; dyphylline 0.15 ; 2-(dimethylamino)-I,3dimethyltetrahydro-1,3,2-diazaphosphole-2-oxid 0.27 ; I,2,5a,6,7,8-hexahydro-6,6-dimethyl-cyclopenta[c]pentalen-3(3aH)-one 0.25 ; alpha-D-glucopyranoside 0.24 ; I-octadecanamine 0.14;2,2-dibutyl-I,2-selenagermolane 0.13; 2-(methylamino)-ethanol 0.09.

Citation: Ivankin AN, Semenova AA, Nasonova VV, et al. Biotechnology for formation of aromatic properties of national- foodstuffs on the basis of meat raw material under influence of bacterial crops and chromato-mass-spectrometric analysis of the flavoring components. J Appl Biotechnol Bioeng. 20I7;3(4):366-372. DOI: I0.15406/jabb.2017.03.00072 
Biotechnology for formation of aromatic properties of national- foodstuffs on the basis of meat raw material under influence of bacterial crops and chromato-mass-spectrometric analysis of the flavoring components

Table Continued..

2-methoxy-phenol 5.29; methoxy-phenyl-oxime 0.37; malic acid 0.20; 2-methoxy-4-methyl-phenol I.02; hexadecane 0.60; I-bromodocosane 0.10; tetradecane 0.54; I-methyl-4-(I-methylethyl)-I,3-cyclohexadiene 0.62;2,6-dimethoxy-phenol I.03; 4-methoxy-3-(methoxymethyl)-phenol 0.78; heneicosane 0.95; 7-hexyl-tridecane I.63; 6-methyl-tridecane 0.93; 5-methoxy-4-methyl-I-heptene 0.20; hentriacontane I.19; hexadecane 0.88; 3,6-dimethyl-undecane 0.32; ethyl tridecanoate I.99; 5-hydroxy2-methyl-3-hexenoic acid 0.07; propyl-2-ethylhexanoate 0.57; 9-hydroxy-2-nonanone 0.2I; n-propyl-9-tetradecenoate I.82; hexadecanoic acid, methyl ester 0.92; hexadecanoic acid, ethyl ester 21.4I;3,5-dinitro-benzonitrile 0.05; decanoic acid, methyl ester 0.23; I-hexadecanol 2.2I; eicosane 0.2I; ethyl oleate 28.25; heptadecanoic acid, ethyl ester 2.I4; octadecanoic acid, ethyl ester 8.96; octahydro-2-methylene-4,7-methano-IH-indene 0.20; nonadecane 0.83; octahydro-4a,5-dimethyl-3-(Imethylethyl)-2(IH)-naphthalenone I.04.

2-methoxy-phenol 6.4I; 6-ethoxy-pyridin-2-amine 0.33 ; 2-methoxy-4-methyl-phenol 3.33; D,L-arabinose 0.27 ; I-iodo-tridecane 0.57 ; 4-ethyl-2-methoxy-phenol I.42; 3-methyl-5-propyl-nonane 0.28; (+)-4-carene 0.54; 2,6-dimethoxy-phenol 0.98; 4-aminobutanoic acid 0.2I; 4-methoxy-2-methyl-I-(methylthio)benzene 0.65; heptadecane I.07; octadecane 8. I8; 5,5-dimethyl-3-(3-methyl-oxiran-2-yl)-cyclohex-2-enone 0.19; N-cyclohexyl-3-nitro-4-pyridinamine 0.60; 3,5-dichloro-2,6-dimethyl4-pyridyl ester 0.12; 2-bromo dodecane I.08; docosane $0.7 \mathrm{I}$; nonanoic acid, ethyl ester I.95; 2-methyl-propanamide 0.4; 2,4,6,8-tetramethyl-I3-tetradecenoic acid 0.5 2-dodecanone 0.4; heneicosane 0.89; methyl hexadec-9-enoate 2.02; hexadecanoic acid, ethyl ester 19.26; 2-(p-tolyl)ethylamine 0.08; 9-eicosene 2.62; 2-methyl-decane 0.27; ethyl oleate 28.53; octadecanoic acid, ethyl ester 16.08; 5-acetoxypentadecane 2.I0; 2,2-dimethyl-5-methylene-bicyclo[2.2. I] heptane 0.23; Z-7-hexadecenoic acid I.12; eicosane I.00; 3,3-dimethyl-2-(phenylselenyl)butanoic acid, 2-methylbutyl ester 0.88 .

2-methoxy-phenol 3.6I; 2-methoxy-4-methyl-phenol 0.9I; tetradecane 0.4I; 4-ethyl-2-methoxy-phenol 0.72; 4-amino-5-imidazole carboxamide 0.20; hentriacontane 0.35; (+)-4-carene 0.53; 2,6-dimethoxy-phenol I.00; 2-methoxy-5-nitro-benzenamine I.I5; hexadecane 0.3; octadecane I.I2; N-acetyl-dl-serine, methyl ester 0.44; 3-chloro-I,2-propanediol 0.07; 4-phenyl-pyrimidine 0.01 ; eicosane 0.67 ; octacosane 0.56 ; 3,6-dimethyl-undecane 0.18 ; tetradecanoic acid, ethyl ester I.45; $\mathrm{N}$-allyl-oxalic acid, monoamide 0.39; 2-pentadecanone 0.36; octacosane 0.62; E-II-hexadecenoic acid I.79; hexadecanoic acid 0.12; cyclohexadecane 2.35; 2-methyl-decanoic acid 0.19; eicosane 0.I2; 9-octadecenoic acid 30.69; octadecanoic acid I7.85; I5-hydroxypentadecanoic acid 2.84; Z,Z,Z-I,4,6,9-nonadecatetraene 0.22; I-cyclohexyInonene 0.8I; octadecane 0.70; N-ethyl-ethanamine 0.2I; I3-octadecenal I.56; 3-(dimethylamino)-2-propenoic acid, methyl ester 0.74; 3,7-dimethyl-2-octen-I-ol 0.I5; benzo(a)pyrene-6-methanol 0.07; 17-hydroxy-pregna-I,4-diene-3,20-dione 0.07; 4-methoxy-6-morpholin-4-yl-[I,3,5]triazine-2-carboxylic acid amide 0.I0; methy 3-diethylphosphonoacrylate $0.10 ; 6$-(2-formylhydrazino)-N,N'-bis(isopropyl)-I,3,5-triazine-2,4-diamine 0.13 ; benzothiophene-3-carboxylic acid 0.10 ; 3-hydroxy4-methoxybenzyl alcohol 0.03 ; I,I'-binaphthalene 0.05 ; 2-hydroxy-IH-isoindole-I,3(2H)-dione 0.06 ; I-azido-2-nitro-benzene 0.08 ; I-adamantanecarboxanilide 0.10 ; II, I2-dihydroxyseychellane 0.07 ; I',2'-epoxyhexobarbital 0.04 ; 2-methylaminomethyl-1,3-dioxolane 0.12 ; 2-acetylamino-3-(4-ethoxy-phenyl)-acrylic acid 0.10; 4-phenyl-3,4-dihydroisoquinoline 0.08; $\mathrm{N}$-methyl-I-adamantaneacetamide 0.12; I-acetyl-4-[I-piperidyl]-2-butynone 0.05; 2-(benzylideneamino)fluorene 0.04; 2-nitrobenzaldehyde 0.1 I; N-(2'-acetyl-4',5'-dimethoxyphenyl)-4-methoxy-benzamide 0.05; 3,4-dimethyl-N-(4-methylthiobenzylidene)-benzenamine 0.09; hexahydropyridine 0.06; IH-pyrazolobisthiolium 0.13; 2-hexadecyl-2,3-dihydro-I H-indene 0.09; 4-[N-methylpiperazino]-5-nitro veratrole 0.05; 7-chloroquinoline-2,4-dicarboxylic acid 0.08; 2-acetylamino-3-(4-ethoxy-phenyl)-acrylic acid 0.08; 3,5-dibromo-4-pyridinol 0.I2; 3-O-methyl-D-glucose 0.07; 8-hydro-thiazolo[3,2-a]pyridinium 0.05; p,p'dibromodiphenyl trichloroethane 0.09; 6-chloro-2-phenethyl-4-phenylquinoline 0.08; I-amino-2-(hydroxymethyl)anthraquinone 0.2; 3-(4-aminophenyl)-2-phenyl-acrylic acid, methyl ester 0.03 ; benzo[b]thiophene-4-acetic acid 0.08; 2 -isopropylidenehydrazono-3-methyl-4-chloro-2,3-dihydrobenzothiazole 0. I0; $\mathrm{N}$-(diphenylethenylidene)methanamine 0.05 ; I,2,4-Oxadiazole-5-carboxamide 0.05 ; pentachloro-pyridine 0.07 ; propanamide 0.03 ; N-methyl-4-pyridinecarboxamide 0.13 ; 4-acetamido-2methallylphenol 0.07; 6-chloro-3-ethoxycarbonyl-2-methyl-4-phenylquinoline 0.07; 4-methylthiophene-3-sulfonamide 0.04; 2,2-diphenylpropionitrile 0.08; I-(5-bromo4-nitro-2-thienyl)ethan-I-one 0.08

IA: Minced meat in a shell with Lactobacillus plantarum+Staphylococcus carnosus.

IB:With cultures of Lactobacillus plantarum+Micrococcus varians.

IC: Minced control in a shell without cultures.

A, B, C: Respectively fermented sausages subjected to smoked.

In Table 2 gives the main "flavor components" of all fermented sausages in comparison with the control. The composition of the present flavoring components mainly includes fat substances fatty acid derivatives, which in the composition are several times larger than other substances from the same but fat-free samples (Table 1). Obtained data seem to suggest that the taste of the national product of Russia - sausage Braunschweigskaya is determined mainly by a combination of basic substances repeated in all samples (Table 2), first of all, the derivatives of fatty acids-decane, hexadecane, 9-octadecene, palmitic , Docosanic, 8,11,14,17-eicosatetraenoic, cis10-nonenedecene, nonane, dodecanoic, tetradecanoic, cholesterol derivatives and impurities of all other components of flavoring substances 1 and 2, involved in the "composition" of the taste and flavor of sausage Braunschweig. The formation of a flavor-aromatic scale occurs due to the spices used and the enzymatic activity of microbial cultures that break down the constituent parts of meat raw materials ${ }^{13-15}$ into organic substances with a characteristic flavor.

Table 2 Basic fragrances of sausages of Braunschweig, $\mathrm{mg} / \mathrm{kg}$

\begin{tabular}{|c|c|c|c|c|}
\hline Characteristic Peak Identification Time, min & Name of Fragrance & A & B & C \\
\hline 4.117 & 3-phenyl-IH-quinolin-2-one & - & - & 0.12 \\
\hline 4.175 & I-ethyl-2-phenyl-IH-indole & - & 0.51 & - \\
\hline 4,631 & Decanoic acid, methyl ester & 0.06 & 0.32 & 0.26 \\
\hline 4.683 & Nonanoic acid, methyl ester & $0.4 \mathrm{I}$ & 1.33 & 1.53 \\
\hline 6.006 & Dodecanoic acid, methyl ester & 0.57 & 1.64 & 1.89 \\
\hline
\end{tabular}

Citation: Ivankin AN, Semenova AA, Nasonova VV, et al. Biotechnology for formation of aromatic properties of national- foodstuffs on the basis of meat raw material under influence of bacterial crops and chromato-mass-spectrometric analysis of the flavoring components. J Appl Biotechnol Bioeng. 20I7;3(4):366-372. DOI: I0.15406/jabb.2017.03.00072 
Table Continued..

\begin{tabular}{|c|c|c|c|c|}
\hline Characteristic Peak Identification Time, min & Name of Fragrance & A & B & C \\
\hline 7.215 & Methyl tetradecanoate & 7.78 & 6.57 & 7.89 \\
\hline 7.729 & Pentadecanoic acid, methyl ester & 0.48 & - & - \\
\hline 8.149 & 9-hexadecenoic acid & - & - & 3.21 \\
\hline 8.175 & Methyl hexadec-9-enoate & 9.42 & 5.49 & - \\
\hline 8.274 & Hexadecanoic acid, methyl ester & 13.47 & 19.53 & 12.19 \\
\hline 8.689 & Methyl cis-10-Heptadecenoate & 2.32 & - & - \\
\hline 8.772 & Heptadecanoic acid, methyl ester & 3.35 & - & - \\
\hline 9.15 & Methyl (Z)-9-Octadecenoate & 26 & 18.96 & 14.79 \\
\hline 9.669 & Methyl cis-10-Nonadecenoate & 1.13 & 4.69 & 0.4 \\
\hline 9.918 & 5,8, II, I4-Eicosatetraenoic acid & 2.81 & - & - \\
\hline 9.97 & cis-5,8, I I-Eicosatrienoic acid & 1.65 & - & - \\
\hline 9.981 & Octadecanoic acid, methyl ester & - & - & 0.36 \\
\hline 10.027 & Octadec-9-en-I-al dimethyl acetal & - & - & 2.7 \\
\hline 10.053 & Z-6, I7-octadecadien-I-ol acetate & - & 2.48 & - \\
\hline 10.074 & 2-octyl-cyclopropaneoctanoic acid & - & - & 4.8 \\
\hline 10.079 & Methyl cis-I3-eicosenoate & 12.04 & - & - \\
\hline 10.136 & Eicosanoic acid & 1.85 & - & 5.78 \\
\hline 10.147 & 3,7, I I-trimethyl-2,6, I0-dodecatrien-I-ol & - & - & 0.46 \\
\hline 10.173 & 2,4-dinitro-5-fluoroaniline & - & 0.72 & - \\
\hline 10.235 & Arachidonic acid & - & 6.65 & - \\
\hline 10.25 & Methyl 7, I0, I3-Eicosatrienoate & - & - & 3.89 \\
\hline 10.276 & I,9-cyclohexadecadiene & - & 3.17 & - \\
\hline 10.282 & 5-nonadecen-I-ol & - & - & 10.46 \\
\hline 10.307 & II-hexadecen-I-ol, acetate & - & 6.66 & - \\
\hline 10.411 & 6-octadecenoic acid, methyl ester & - & - & 1.16 \\
\hline 10.416 & Methyl I8-methylnonadecanoate & - & 3.35 & - \\
\hline 10.697 & Methyl 8, II, I4, I7-eicosatetraenoat & 0.61 & 0.4 & 3.73 \\
\hline 10.774 & 9-octadecenal & 4.46 & - & - \\
\hline 10.832 & Ethyl 5,8, I I, I4, I7-icosapentaenoat & - & 4.11 & 7.23 \\
\hline 10.847 & Palmitoyl chloride & 1.87 & 0.15 & 0.19 \\
\hline 10.883 & 2,3-dihydroxypropyl elaidate & - & - & 8.27 \\
\hline 10.889 & I-cyclohexylnonene & - & 6.67 & - \\
\hline 10.925 & Docosanoic acid & 0.27 & 0.56 & 0.82 \\
\hline 10.951 & D-gulopyranoside & - & 2.59 & 2.99 \\
\hline 11.791 & I0-nonadecenoic acid, methyl ester & - & - & 0.32 \\
\hline 11.885 & Tetracosanoic acid, methyl ester & - & - & 0.17 \\
\hline 15.382 & Cholesterol & 0.1 & $0.4 I$ & 0.63 \\
\hline 20.296 & I-docosene & 0.11 & - & - \\
\hline 20.301 & Octacosyl acetate & - & 0.21 & - \\
\hline 20.306 & |3-tetradecen-I-ol acetate & - & - & 0.24 \\
\hline
\end{tabular}

A: Sausage product with Lactobacillus plantarum +Staphylococcus carnosus.

B: Sausage product with cultures of Lactobacillus plantarum +Micrococcus varians.

C: Control without crops.

Citation: Ivankin AN, Semenova AA, Nasonova VV, et al. Biotechnology for formation of aromatic properties of national- foodstuffs on the basis of meat raw material under influence of bacterial crops and chromato-mass-spectrometric analysis of the flavoring components. J Appl Biotechnol Bioeng. 20I7;3(4):366-372. DOI: I0.15406/jabb.2017.03.00072 


\section{Conclusion}

Thus, the targeted introduction of certain microbial cultures allows us to adjust the flavor and aroma of national meat products, due to the presence of traditional spices.

\section{Acknowledgements}

None.

\section{Conflict of interest}

The author declares no conflict of interest.

\section{References}

1. Grin AI, Vysotskaya LE, Mikhailova TV. Chemistry of taste and smell of meat products. Ukraine: Kiev: Naukova Dumka; 1985.

2. Khankhalaeva IA, Khamaganova IV. Influence of starter cultures on the taste and aroma of raw sausage sausages. Meat Industry (Russ). 2008;3:53-55.

3. Stahnke LH, Sunesen LO, Smedt AD. Sensory characteristics of European, dried, fermented sausages and the correlation to the volatile profile/In Proceedings of the $13^{\text {th }}$ Forum for Applied Biotechnology. Mededelingen Faculteit Landbouwkundige en Toegepaste Biologische Wetenschapppen, Russia; 1999. p. 559-566.

4. Mateo J, Zumalacárregui J. Volatile compounds in Chorizo and their changes during ripening. Meat Sci. 1996;44(4):255-273.

5. Sunesen LO, Dorigoni V, Zanardi E, et al. Volatile compounds released during ripening in Italian dried sausage. Meat Science. 2001;58(1):9397.

6. Vandekerckhove P, Demeyer D. The composition of the Belgian dry sausage (salami). Fleischwirtsch. 1975;55:680-682.

7. Casaburi A, Aristoy MC, Cavella S, et al. Biochemical and sensory characteristics of traditional fermented sausages of Vallo di Diano (Southern Italy) as affected by the use of starter cultures. Meat Sci. 2007;76(2):295-307.

8. Partidário AM, Padilha M, Roseiro C, et al. Volatile compounds produced during ripening of the Portinho de Portalegre dry fermented sausage. Revista Portuguesa de Ciências Veterinárias Rpcv. 2006;101(1):115-120.

9. Hua ZL. Study on the Quality Characteristics and Changes in Formation Mechanism of Volatile Flavor of Mutton Fermented Dry Sausage. China: Inner Mongolia Agricultural University; 2009.

10. Gianelli MP, Salazar V, Mojica L, et al. Volatile compounds present in traditional meat products (charqui and longaniza sausage) in Chile. Braz arch biol Technol. 2016;55(4).

11. Lisitsyn AB, Ivankin AN, Neklyudov AD. Methods of practical biotechnology. Moscow: Russia: VNIIMP; 2012.

12. Zagustina NA, Misharina TA, Veprizky AA, et al. Elimination of volatile compounds of leaf tobacco from air emissions using biofiltration. Appl Biochem Microbiol. 2012;48(4):385-395.

13. Ivankin AN. Fats in the composition of modern meat products. Meat industry (Russ). 2007;6:8-13.

14. Neklyudov AD, Ivankin AN. Biochemical processing of fats and oils as a means of obtaining lipid products with improved biological and physicochemical properties: a review. Applied Biochemistry and Microbiology. 2002;38(5):399-409.
15. Neklyudov AD, Ivankin AN, Berdutina AV. Properties and uses of protein hydrolysates. Appl Biochem Microbiol. 2000;36(5):452-459.

16. Bohr A, Boetker J, Wang Y, et al. High-throughput fabrication of nanocomplexes using 3D-printed micromixers. J Pharm Sci. 2017;106(3):835-842.

17. Chen X, Li T, Zeng H, et al. Numerical and experimental investigation on micromixers with serpentine microchannels. International Journal of Heat and Mass Transfer. 2016;98(7):131-140.

18. Chen X, Li T. A novel design for passive misscromixers based on topology optimization method. Biomed Microdevices. 2016;18(4):57.

19. Rafeie M, Welleweerd M, Barforoushi AH, et al. An easily fabricated three-dimensional threaded lemniscate-shaped micromixer for a wide range of flow rates. Biomicrofluidics. 2017;11(1):014108.

20. Chen X, Li T. A novel passive micromixer designed by applying an optimization algorithm to the zigzag microchannel. Chemical Engineering Journal. 2017;313(4):1406-1414.

21. Chen X, Liu C, Xu Z, et al. Macro-micro modeling design in system-level and experiment for a micromixer. Analytical Methods. 2012;4:2334.

22. Yildirim E. Analysis and testing of a contraction-and-expansion micromixer for micromilled microfluidics. Microsyst Technol. 2017;1:1-8.

23. Chen X, Shen J. Simulation in system-level based on model order reduction for a square wave micromixer. International Journal of Nonlinear Sciences and Numerical Simulation. 2015;16(7-8).

24. Chen X, Wang X. Optimized modular design and experiment for staggered herringbone chaotic micromixer. International Journal of Chemical Reactor Engineering. 2015;13(3).

25. Xie T, Xu C. Numerical and experimental investigations of chaotic mixing behavior in an oscillating feedback micromixer. Chemical Engineering Science. 2017;171(2):303-317.

26. Chen X, Li T, Li X. Numerical research on shape optimization of microchannels of passive micromixers. IEEE Sensors Journal. 2016;16(17):6527-6532.

27. Chen X, Zhang Z, Yi D, et al. Numerical studies on different two-dimensional micromixers basing on a fractal-like tree network. Microsyst Technol. 2017;23(3):755-763.

28. Chen X, Shen J. Design and simulation of a chaotic micromixer with diamond-like micropillar based on artificial neural network. International Journal of Chemical Reactor Engineering. 2016;15(2).

29. Chen X, Shen J. Numerical analysis of mixing behaviors of two types of E-shape micromixers. International Journal of Heat and Mass Transfer. 2017;106(3):593-600.

30. Mierka O, Munir M, Spille C, et al. Reactive liquid-flow simulation of micromixers based on grid deformation techniques. Chem Eng Technol. 2017;3:201600686.

31. Chen X, Liu C, Xu Z, et al. An effective PDMS microfluidic chip for chemiluminescence detection of cobalt (II) in water. Microsyst Technol. 203;19(1):99-103.

32. Chen X, Shen J. Numerical and experimental investigation on splitting-and-recombination micromixer with E-shape mixing units. $\mathrm{Mi}$ crosyst Technol. 2016;7:1-7.

33. Huang CY, Wan SA, Hu YH. Oxygen and nitrogen gases mixing in T-type micromixers visualized and quantitatively characterized using pressure-sensitive paint. International Journal of Heat and Mass Transfer. 2017;111(8):520-531. 